\title{
Spectral energy transfer of atmospheric gravity waves through sum and difference nonlinear interactions
}

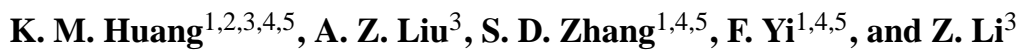 \\ ${ }^{1}$ School of Electronic Information, Wuhan University, Wuhan, China \\ ${ }^{2}$ Key Laboratory of Geospace Environment, University of Science \& Technology of China, \\ Chinese Academy of Sciences, Hefei, China \\ ${ }^{3}$ Department of Physical Science, Embry Riddle Aeronautical University, Daytona Beach, Florida, USA \\ ${ }^{4}$ Key Laboratory of Geospace Environment and Geodesy, Ministry of Education, Wuhan, China \\ ${ }^{5}$ State Observatory for Atmospheric Remote Sensing, Wuhan, China
}

Correspondence to: S. D. Zhang (zsd@whu.edu.cn), K. M. Huang (hkm@whu.edu.cn)

Received: 5 October 2011 - Revised: 20 December 2011 - Accepted: 22 December 2011 - Published: 3 February 2012

\begin{abstract}
Nonlinear interactions of gravity waves are studied with a two-dimensional, fully nonlinear model. The energy exchanges among resonant and near-resonant triads are examined in order to understand the spectral energy transfer through interactions. The results show that in both resonant and near-resonant interactions, the energy exchange between two high frequency waves is strong, but the energy transfer from large to small vertical scale waves is rather weak. This suggests that the energy cascade toward large vertical wavenumbers through nonlinear interaction is inefficient, which is different from the rapid turbulence cascade. Because of considerable energy exchange, nonlinear interactions can effectively spread high frequency spectrum, and play a significant role in limiting wave amplitude growth and transferring energy into higher altitudes. In resonant interaction, the interacting waves obey the resonant matching conditions, and resonant excitation is reversible, while near-resonant excitation is not so. Although near-resonant interaction shows the complexity of match relation, numerical experiments show an interesting result that when sum and difference near-resonant interactions occur between high and low frequency waves, the wave vectors tend to approximately match in horizontal direction, and the frequency of the excited waves is also close to the matching value.
\end{abstract}

Keywords. Meteorology and atmospheric dynamics (Middle atmosphere dynamics)

\section{Introduction}

Gravity waves are one of the most important waves in the middle and upper atmosphere and play a crucial role in determining large-scale circulation and thermal structure of the middle atmosphere because of their inherent ability to transport momentum and energy from one atmospheric layer to another (Lindzen, 1981; Holton, 1982; Vincent and Reid 1983; Fritts and Dunkerton, 1985). Gravity wave activity has been statistically investigated based on various observations (Tsuda et al., 1990; Manson and Meek, 1993; Nekrasov et at., 1995; Vincent and Alexander, 2000; Zink and Vincent, 2001; Alexander and Teitelbaum, 2007; Zhang and Yi, 2005, 2007; Hei et al., 2008; Zhang et al., 2010), and possible mechanisms of generation and dissipation of gravity waves have been extensively explored in the past decades (Walterscheid and Schubert, 1990; Fovell et al., 1992; Fritts and Luo, 1992; Alexander et al., 1995; Hecht, 2001; Lane et al., 2001; Kshevetskii and Gavrilov, 2005; Liu and Gardner, 2005; Liu, 2009; Vadas and Liu, 2009). Nonlinearity is an important aspect of gravity wave dynamics. Nonlinear interaction of gravity waves can lead to the redistribution of energy and momentum among different spectral components with approximate conservation of total energy and momentum, but does not destroy wave structure as the instability and breaking of waves do. Nonlinear cascade of gravity waves through interaction may lead to turbulences by generating waves with vertical scales so small that they become saturated and broken. Simultaneously, effective energy transfer 
in interaction can limit the amplitude growth of waves, which decreases the probability of these components reaching their instability thresholds, and then their energy and momentum may further be transported into higher levels. When gravity waves with different temporal and spatial scales propagate in the middle and upper atmosphere, they may randomly encounter and superpose. At this time, energy transfer among different spectral components may frequently happen through nonlinear interaction, which may result in rapid relaxation of distorted spectra toward the universal form of gravity waves. Broutman et al. (1997) used numerical simulations to examine the Doppler spread of a short wave packet interacting with a single inertia wave packet, and showed that the spectrum developed into a broad region with slope of about -2.2 with two inertia periods. Therefore, wavewave interaction is regarded to be not only a significant wave source in the middle and upper atmosphere (Fritts and Alexander, 2003), but also an important mechanism in spectral evolution for gravity waves in the middle and upper atmosphere (Weinstock, 1985; Yeh and Liu, 1985; Smith et al., 1987; Hines, 1991, 2001; Dewan, 1994; Chunchuzov, 2009).

The phenomenon of resonant interaction in a fluid dynamical context was discovered by Phillips (1960). He pointed out that for prescribed wave vectors $\boldsymbol{k}$ and frequencies $\omega$, two waves with phases $\boldsymbol{k}_{1} \cdot \boldsymbol{r}-\omega_{1} t$ and $\boldsymbol{k}_{2} \cdot \boldsymbol{r}-\omega_{2} t$ can act as forcing terms in the linearized equations to force a third wave with their sum or difference phase. If the third wave is a free mode, resonant interaction occurs and significant energy transfer among the wave triad takes place. The resonant conditions are expressed as

$\boldsymbol{k}_{1} \pm \boldsymbol{k}_{2}=\boldsymbol{k}_{3}$

$\omega_{1} \pm \omega_{2}=\omega_{3}$

where the subscripts $j=1,2$ and 3 denote the interacting three waves; $\omega_{j}=\Omega\left(\boldsymbol{k}_{j}\right)$ is the dispersion relation, which can be written as $\frac{k_{x}^{2}}{\left(\omega^{2}-\omega_{\mathrm{a}}^{2}\right) /\left(\omega^{2}-N^{2}\right)}+\frac{k_{z}^{2}}{\left(1-\omega_{\mathrm{a}}^{2} / \omega^{2}\right)}=\frac{\omega^{2}}{v_{\mathrm{a}}^{2}} ; k_{x}$ and $k_{z}$ are the horizontal and vertical components of the wave vectors, respectively; $N$ is the buoyancy frequency; $v_{\mathrm{a}}$ is the acoustic speed; and $\omega_{\mathrm{a}}$ is the acoustic-cutoff frequency. Considering that two initial waves may play different roles, the high frequency wave is named as the primary wave and the other one with low frequency is regarded as the secondary wave. In the following numerical experiments, the subscripts $j=1,2$ and 3 do not specify the primary, secondary and excited waves, respectively, and the roles of the primary and secondary waves depend on their frequencies in each case. These two types of resonant interactions presented in Eqs. (1) and (2) are referred to as Sum and difference resonant interactions, respectively. Correspondingly, if these interacting waves do not satisfy the resonant matching conditions of Eqs. (1) and (2), nonresonant interaction takes place (Whitham, 1974), and the two kinds of interactions are regarded as sum and difference nonresonant interactions, respectively. When the mismatching magnitude is small, it is also viewed as near-resonant interaction.

The studies on nonlinear interaction of gravity waves have made great advances by the theoretical, observational and modelling efforts. On the basis of the weak interaction approximation, many features and effects of resonant interaction of oceanic internal gravity waves have been acquired (Phillips, 1960; Bretherton, 1964; Hasselmann, 1966; Olbers, 1976; McComas and Bretherton, 1977; McComas and Müller, 1981; Müller et al., 1986). Subsequently, the properties of resonant and nonresonant interactions among atmospheric gravity waves have been extensively investigated (Dysthe et al., 1974; Yeh and Liu, 1981, 1985; Klostermeyer, 1982, 1991; Inhester, 1987; Dong and Yeh, 1988; Yeh and Dong, 1989), even in a sheared, dissipative and rotating atmosphere (Fritts et al., 1992; Axelsson et al., 1996; Yi and Xiao, 1997), and in the uniform and nonuniform plasmas (Stenflo, 1994; Stenflo and Shukla, 2009). These theoretical studies provided essential understanding of nonlinear interactions of gravity waves. Resonant triads in atmospheric gravity wave domain were reported based on radar, rocket and satellite observations (Klostermeyer, 1984, 1990; Widdel et al., 1994; Rüster, 1997; Wüst and Bittner, 2006). Atmospheric radar observations also presented extensive evidences of resonant interactions among atmospheric waves (Rüster, 1994; Manson et al., 1998; Beard et al., 1999; Pancheva, 2001; She et al., 2004). Many model works further confirmed three-wave resonant and nonresonant interactions in the middle and upper atmosphere (Dunkerton, 1987; Vanneste, 1995; Yi, 1999; Zhang and Yi, 2004; Huang et al., 2007, 2009). The numerical studies clarified that energy transfer in nonlinear interaction from the high frequency primary wave to the excited wave is irreversible rather than periodic from the weak interaction approximation, and significant energy exchange in nonresonant interaction may be comparable to that in resonant interaction (Zhang and Yi, 2004; Huang et al., 2007, 2009).

However, most numerical studies, mentioned above, focused on a certain sum or difference interaction and some important characteristics of nonlinear interactions may not be revealed only by the investigation on a single nonlinear excitation. Thus, there remains much to be done in quantitative studies on the dynamics and effects of nonlinear interactions of atmospheric gravity waves (Fritts and Alexander, 2003). In this paper, sum and difference nonlinear interactions of gravity waves are simulated and the features of resonant and near-resonant triad interactions are investigated. By analysing the characteristic of energy transfer among spectral components, we will explore the role of nonlinear cascade in gravity wave dissipation and the effect of nonlinear interaction to spectral broadening.

We begin in Sect. 2 with a brief description about the full nonlinear numerical model. In Sect. 3, we exhibit sum and difference resonant excitations for a resonant triad and 
Table 1. Initial parameters of waves 1-3 regarded as initial perturbations in cases 1-3.

\begin{tabular}{rrrcrrrrr}
\hline & $\begin{array}{r}\lambda_{x} \\
(\mathrm{~km})\end{array}$ & $\begin{array}{r}\lambda_{z} \\
(\mathrm{~km})\end{array}$ & $\begin{array}{c}k_{x} \\
\left(10^{-4} \mathrm{Rad} \mathrm{m}^{-1}\right)\end{array}$ & $\begin{array}{r}k_{z} \\
\left(10^{-3} \mathrm{Rad} \mathrm{m}^{-1}\right)\end{array}$ & $\begin{array}{r}\omega \\
\left(10^{-4} \mathrm{Rad} \mathrm{s}^{-1}\right)\end{array}$ & $\begin{array}{c}u_{c} \\
\left(\mathrm{~ms}^{-1}\right)\end{array}$ & $\begin{array}{c}x_{c} \\
(\mathrm{~km})\end{array}$ & $\begin{array}{c}z_{c} \\
(\mathrm{~km})\end{array}$ \\
\hline Wave 1 & 50 & 5.0 & 1.26 & 1.26 & 18.05 & 5.0 & 500 & 80 \\
Wave 2 & 129.67 & -2.3 & 0.48 & -2.73 & 3.22 & 1.0 & 600 & 60 \\
Wave 3 & 36.08 & -4.26 & 1.74 & -1.47 & 21.27 & 0.4 & 530 & 43 \\
\hline
\end{tabular}

quantitatively analyse the energy exchange magnitude. In Sect. 4, several near-resonant triads are performed to reveal the general characteristics of energy exchange in sum and difference nonlinear interactions. The summary is provided in Sect. 5.

\section{Numerical model}

\subsection{Governing equations and numerical scheme}

The simulations use a set of primitive hydrodynamic equations in an adiabatic, inviscid and two-dimensional compressible atmosphere, which can be written as

$$
\left\{\begin{array}{l}
\frac{\partial \rho}{\partial t}+u \frac{\partial \rho}{\partial x}+w \frac{\partial \rho}{\partial z}+\rho \frac{\partial u}{\partial x}+\rho \frac{\partial w}{\partial z}=0 \\
\frac{\partial u}{\partial t}+u \frac{\partial u}{\partial x}+w \frac{\partial u}{\partial z}+\frac{R T}{\rho} \frac{\partial \rho}{\partial x}+R \frac{\partial T}{\partial x}=0 \\
\frac{\partial w}{\partial t}+u \frac{\partial w}{\partial x}+w \frac{\partial w}{\partial z}+\frac{R T}{\rho} \frac{\partial \rho}{\partial z}+R \frac{\partial T}{\partial z}+g=0 \\
\frac{\partial T}{\partial t}+u \frac{\partial T}{\partial x}+w \frac{\partial T}{\partial z}+T(\gamma-1)\left(\frac{\partial u}{\partial x}+\frac{\partial w}{\partial z}\right)=0
\end{array}\right.
$$

Where $x$ and $z$ are the horizontal and vertical (positive upward) coordinates, respectively; $u$ and $w$ are the horizontal and vertical components of total wind velocities, respectively; $\rho$ and $T$ are the density and temperature, respectively; $g$ is the gravitational acceleration; $R=287 \mathrm{~J} \mathrm{~kg}^{-1} \mathrm{~K}^{-1}$ is the special gas constant for air; and $\gamma=c_{p} / c_{v}\left(c_{p}=\right.$ $1005 \mathrm{~J} \mathrm{~kg}^{-1} \mathrm{~K}^{-1}$ and $c_{v}=718 \mathrm{~J} \mathrm{~kg}^{-1} \mathrm{~K}^{-1}$ are specific heats at constant pressure and volume, respectively).

Usually, propagation of gravity waves in the atmosphere is a long-lasting process. For the sake of precisely simulating the propagation and interaction of gravity waves, a numerical scheme should be of high accuracy and fine stability. Here, a composite difference scheme with second-order temporal and spatial precisions is applied to discretizing the Eq. (3), which is described in detail by Huang et al. (2007), and a three-dimensional model was extended to investigate the propagation characteristics of gravity waves (Huang et al., 2008). A uniform Eulerian mesh with staggered grids is applied in order to eliminate the checkerboard error $(\mathrm{Hu}$ et al., 2003). To avoid boundary reflection, the lateral boundaries are set to be periodical and projected characteristic line boundaries are employed at the top and bottom boundaries (Hu and $\mathrm{Wu}, 1984$; Zhang and Yi, 1999).
In view of an explicit scheme applied in the projected characteristic line boundaries, the time step should be restricted by the Courant condition (Hirsch, 1990):

$$
\Delta t<\Delta t_{c}=\frac{1}{\left(v_{\mathrm{a}}+v\right)\left(\frac{1}{\Delta x^{2}}+\frac{1}{\Delta z^{2}}\right)^{\frac{1}{2}}}
$$

where the acoustic speed $v_{\mathrm{a}}=\sqrt{\gamma R T}$ and $v=\sqrt{u^{2}+w^{2}}$. We take $\Delta t=0.5 \Delta t_{c}$ in this paper.

\subsection{Initial background and perturbation}

In order to investigate the relationships among the wavenumbers and frequencies of the interacting gravity waves, we avoid the influences of background wind and inhomogeneous temperature fields by assuming that the initial background atmosphere is windless and isothermal and is in hydrostatic equilibrium with initial constant temperature of $T_{0}=290 \mathrm{~K}$ and initial density profile of $\rho_{0}=\rho_{c} e^{-g z / R T_{0}}$, where $\rho_{c}=$ $1.2 \mathrm{~kg} \mathrm{~m}^{-3}$ is the density at the ground. The buoyancy frequency is calculated to be $N=1.82 \times 10^{-2} \mathrm{~Hz}$.

In the initial background atmosphere, we introduce two discrete gravity wave packets as the initial wave perturbations, for which the horizontal velocity disturbances have the following forms

$$
\begin{gathered}
\left.u_{j}^{\prime}(x, z, t)\right|_{t=0}=u_{c j} e^{-\frac{\left(x-x_{c j}\right)^{2}}{2 \delta_{x j}^{2}}} e^{-\frac{\left(z-z_{c j}\right)^{2}}{2 \delta_{z j}^{2}}} \\
\sin \left[k_{x j}\left(x-x_{c j}\right)+k_{z j}\left(z-z_{c j}\right)-\omega t\right]
\end{gathered}
$$

where $u_{c j}(j=1$ and 2$)$ is the maximum horizontal wind amplitude; $x_{c j}$ and $z_{c j}$ are the initial geometric center positions of the wave packets in the horizontal and vertical directions, respectively; $\sigma_{x j}$ and $\sigma_{z j}$ represent the half-widths of the wave packets in the horizontal and vertical directions, which are chosen to be $\sigma_{x j}=\lambda_{x j}$ and $\sigma_{z j}=\lambda_{z j}$, where $\lambda_{x j}$ and $\lambda_{z j}$ are the horizontal and vertical wavelengths of the interacting waves, respectively.

\section{Sum and difference resonant interactions}

In the first case group (cases 1-3), we design a resonant triad of waves 1-3, of which the wavenumbers and frequencies are listed in Table 1, where the negative sign of the vertical wavelength represents downward phase propagation. In this 

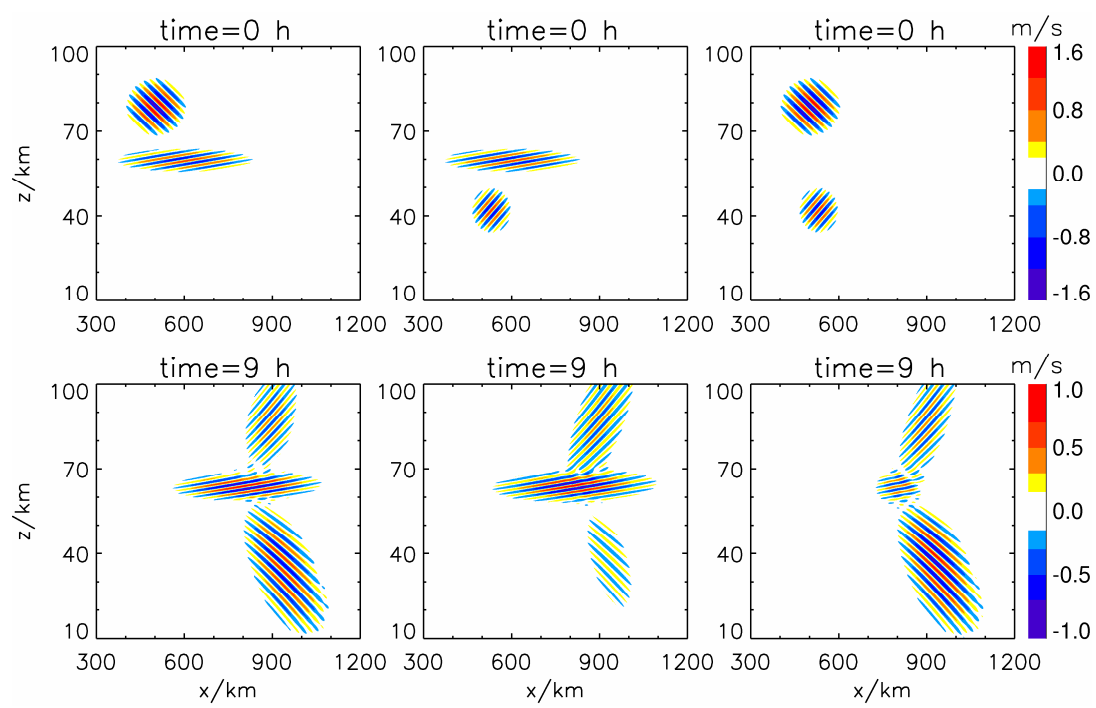

Fig. 1. Sum and difference resonant interactions in cases 1-3. The first, secondary and third columns represent results in cases $1-3$, respectively.

case group, waves 1 and 2, waves 2 and 3, and waves 1 and 3 are selected to be the initial wave perturbations in cases $1-3$, respectively. In the numerical computation, considering the scale of the interacting gravity waves, the grid sizes are set to be $\Delta x=3.6 \mathrm{~km}$ and $\Delta z=0.23 \mathrm{~km}$ in the horizontal and vertical directions, and the computational domains are chosen to be $0 \leq x \leq 2376 \mathrm{~km}$ and $0 \leq z \leq 184 \mathrm{~km}$, respectively. The time step $\Delta t$ can be estimated to be about $0.33-0.34 \mathrm{~s}$ in the computation.

By analysing the wavenumbers and frequencies of waves 1-3 listed Table 1, we may expect that the sum resonant excitation may occur in case 1 due to $\boldsymbol{k}_{1}+\boldsymbol{k}_{2}=\boldsymbol{k}_{3}$ and $\omega_{1}+\omega_{2}=\omega_{3}$, while the difference resonant interaction probably arises in cases 2 and 3 because of $\boldsymbol{k}_{3}-\boldsymbol{k}_{2}=\boldsymbol{k}_{1}$ and $\omega_{3}-\omega_{2}=\omega_{1}$ in case 2 , and $\boldsymbol{k}_{3}-\boldsymbol{k}_{1}=\boldsymbol{k}_{2}$ and $\omega_{3}-\omega_{1}=\omega_{2}$ in case 3 . Since the frequency of the primary wave is higher than that of the secondary wave, the primary wave should be wave 1 in case 1 , and wave 3 in cases 2 and 3 ; and the secondary wave is the other initial wave in each case. When waves 1-3 are regarded as the initial wave perturbations, their amplitudes and centre positions are also listed in Table 1 . This means that as the initial waves, waves 1-3 have the same parameters in the different cases.

Figure 1 exhibits the new wave excitation through the nonlinear interaction in cases 1-3 using the square root densityweighted horizontal velocity disturbances, which are calculated as $u^{\prime \prime}(z)=\left[\rho_{0}(z) / \rho_{0 r}\right]^{\frac{1}{2}} u^{\prime}(z)$, where $\rho_{0 r}$ is the background density at a reference level of $z=60 \mathrm{~km}$. At the beginning time, two separate initial waves are situated at the different heights. After $9 \mathrm{~h}$ of propagation and interaction, a new wave is clearly excited in each case, and these three interacting waves are almost apart from each other.
The wavenumber spectra of three waves are obtained by making a two-dimensional Discrete Fourier Transform (DFT) on $u^{\prime \prime}$ over the whole computational domain. The calculated wavenumber spectra are normalized by the maximum spectral magnitude at the initial time. Figure 2 exhibits the normalized wavenumber spectra of the interacting waves at $t=0$ and $9 \mathrm{~h}$ for cases $1-3$. According to the peak spectrum of the excited wave at the time of $9 \mathrm{~h}$, we calculate the dominant wavelengths of the excited waves. In cases 1-3, the wavelengths of the excited waves in the horizontal and vertical directions are 36.0 and $-4.28 \mathrm{~km}, 50.55$ and $4.97 \mathrm{~km}$, and 125.05 and $-2.30 \mathrm{~km}$, respectively, which are in agreement with the horizontal and vertical matching wavelengths listed in Table 1, respectively. The tiny difference between the calculated and matching wavelengths is due to limited spectral resolution in the numerical computation. Hence, the new waves should be excited through the sum resonant interaction in case 1 and through the difference resonant interaction in cases 2 and 3. Moreover, it can be seen in Fig. 2 that the dominant wavelengths of the primary and secondary waves remain unchanged in the interactions in each case. This means that in the resonant interactions, the three interacting waves satisfy the resonant matching conditions, as predicted by the weak interaction theory. Because the interacting waves abide by the resonant conditions, for the resonant wave triad, if any two waves are chosen to be the initial wave perturbations, the third wave can be excited through the sum or difference resonant interaction, as shown in Figs. 1 and 2. In other words, the resonant excitation exhibits a reversible characteristic.

We discuss the energy exchange among the waves in the resonant interaction. The energies of waves 1-3 at each integral hour are calculated by integrating the wave energy density $\varepsilon_{\mathrm{W}}=\frac{1}{2} \rho_{0}\left(u^{\prime 2}+w^{\prime 2}\right)+\frac{p^{\prime}}{2 \rho_{0} v_{\mathrm{a}}^{2}}+\frac{\left(p^{\prime}-\rho_{0} v_{\mathrm{a}}^{2}\right)}{2 \rho_{0}(\gamma-1) v_{\mathrm{a}}^{2}}$ over the whole 

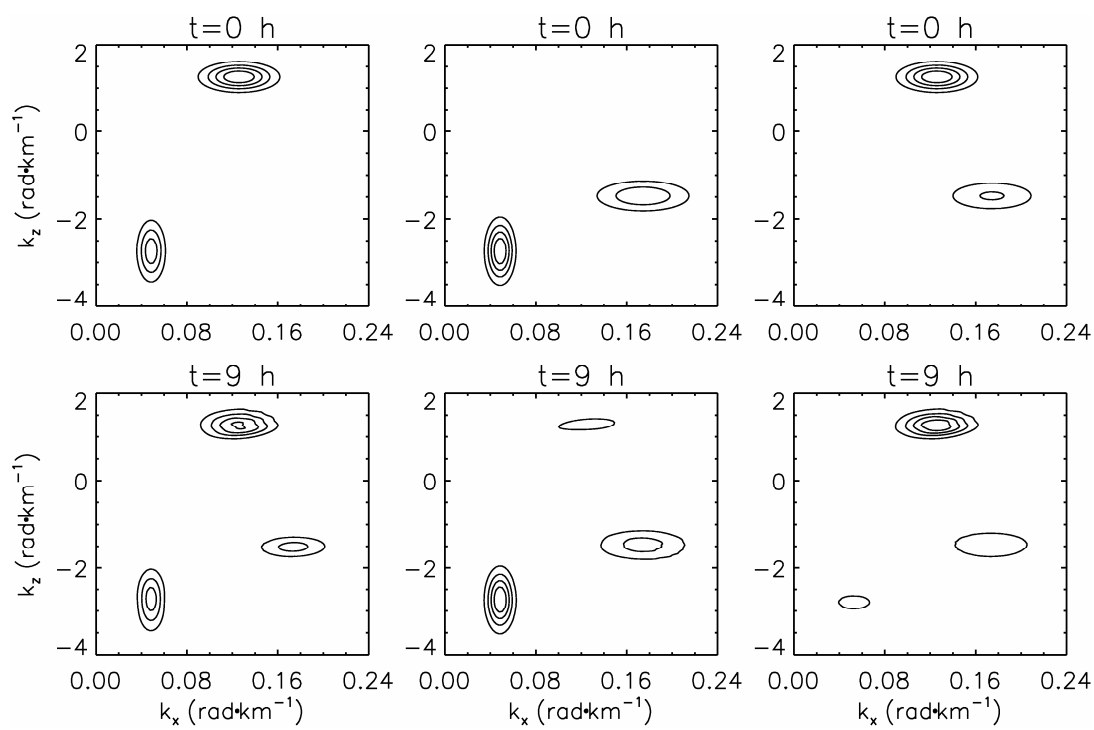

Fig. 2. Normalized wave number spectra of interacting wave triad in cases $1-3$. The contour values are $0.2,04,0.6$ and 0.8 . The first, secondary and third columns represent results in cases $1-3$, respectively.

computational domain, where $p^{\prime}$ is pressure perturbation; $w^{\prime}$ is vertical disturbance velocity; and the perturbation quantities of each wave are extracted from the corresponding total field by applying a band-pass filter since the spectra of the interacting waves are apart from each other, as shown in Fig. 2. Figure 3 shows the temporal evolution of wave energies in cases 1-3. In Fig. 3, the green, yellow, red and blue curves represent the energies of the primary, secondary and excited waves and their sum, respectively. It can be seen from Fig. 3 that from 3 to $6 \mathrm{~h}$, the energies of the primary waves decrease and are transferred to the excited waves. At the beginning time, the energies of waves $1-3$ are $10.25 \times 10^{5} \mathrm{~J}, 4.87 \times 10^{5} \mathrm{~J}$ and $3.01 \times 10^{5} \mathrm{~J}$, respectively. After $9 \mathrm{~h}$, in case 1 , the excited wave obtains the energy of $2.13 \times 10^{5} \mathrm{~J}$ through the nonlinear interaction, which is about $20.78 \%$ of the initial energy of the primary wave (wave 1). As shown in Fig. 3, the wave energy exchange mainly arises between the primary and excited waves in the interactions, therefore, we define the ratio of the energy of the excited wave to the initial energy of the primary wave as the degree of interaction, which is a measure of the interaction strength (Huang et al., 2009). The degree of interaction is $20.78 \%$ in case 1 . In cases 2 , the new wave has the energy of $5.13 \times 10^{4} \mathrm{~J}$, hence, the degree of interaction are calculated to be $17.04 \%$. In case 3 , the new wave can absorb only the energy of $1.28 \times 10^{4} \mathrm{~J}$ in the resonant interaction, which corresponds to a rather small interaction degree of $4.25 \%$. This is an indication that in despite of an identical resonant wave triad, when two different waves of this triad are chosen to be the initial wave perturbations, the magnitude of the energy transfer may be remarkably different.
In terms of the total wave energies, it shows a slight decrease with loss of about 3-4\% in cases 1-3. The small loss of wave energy is attributable to the small wave energy conversion to the large-scale background movement in longlasting nonlinear propagation and the energy loss in the numerical computation and filtering process. Thus, the total energy of these interacting waves is approximately conservative during the interaction.

\section{Sum and difference near-resonant interactions}

In the second case group (cases 4-6), we chose waves 1 and 2 , waves 2 and 3, and waves 1 and 3 to be the initial wave perturbations in cases $4-6$, respectively. The initial parameters of waves 1-3 are presented in Table 2. Because of the variation of the spatial scale of the interacting waves, in the numerical computation, the grid sizes are adjusted to be $\Delta x=4.0 \mathrm{~km}$ and $\Delta z=0.28 \mathrm{~km}$, respectively.

Here, the possible interaction between two initial waves in cases 4-6 is discussed in brief. In case 4, for specified waves 1 and 2 , if an excited wave meets the sum matching condition of wavenumbers, its wavelengths should equal the matching wavelengths, i.e., $\lambda_{x}=L_{s x}=40 \mathrm{~km}$ and $\lambda_{z}=L_{s z}=$ $-6.36 \mathrm{~km}$, and then its frequency is $28.46 \times 10^{-4} \operatorname{Rad~s}^{-1}$ derived from the dispersion relation of gravity waves, which is obviously larger than the sum $\left(20.59 \times 10^{-4} \mathrm{Rad} \mathrm{s}^{-1}\right)$ of the frequencies of waves 1 and 2 . If the interacting three waves obey the difference matching condition for wavenumbers, the wavelengths of the excited waves are calculated to be $\lambda_{x}=L_{d x}=66.67 \mathrm{~km}$ and $\lambda_{z}=L_{d z}=1.79 \mathrm{~km}$, thus, its frequency $\left(4.87 \times 10^{-4} \mathrm{Rad} \mathrm{s}^{-1}\right)$ is far less than the difference $\left(15.51 \times 10^{-4} \operatorname{Rad~s}^{-1}\right)$ of the frequencies of waves 1 
Table 2. Initial parameters of waves 1-3 regarded as initial perturbations in cases 4-6.

\begin{tabular}{lrrcrrrrr}
\hline & $\begin{array}{r}\lambda_{x} \\
(\mathrm{~km})\end{array}$ & $\begin{array}{r}\lambda_{z} \\
(\mathrm{~km})\end{array}$ & $\begin{array}{c}k_{x} \\
\left(10^{-4} \mathrm{Rad} \mathrm{m}^{-1}\right)\end{array}$ & $\begin{array}{r}k_{z} \\
\left(10^{-3} \mathrm{Rad} \mathrm{m}^{-1}\right)\end{array}$ & $\begin{array}{r}\omega \\
\left(10^{-4} \mathrm{Rad} \mathrm{s}^{-1}\right)\end{array}$ & $\begin{array}{c}u_{c} \\
\left(\mathrm{~ms}^{-1}\right)\end{array}$ & $\begin{array}{c}x_{c} \\
(\mathrm{~km})\end{array}$ & $\begin{array}{c}z_{c} \\
(\mathrm{~km})\end{array}$ \\
\hline Wave 1 & 50 & 5.0 & 1.26 & 1.26 & 18.05 & 5.0 & 720 & 80 \\
Wave 2 & 200 & -2.8 & 0.31 & -2.24 & 2.54 & 1.0 & 800 & 60 \\
Wave 3 & 40 & -4.72 & 1.57 & -1.33 & 21.26 & 0.4 & 739 & 43 \\
\hline
\end{tabular}
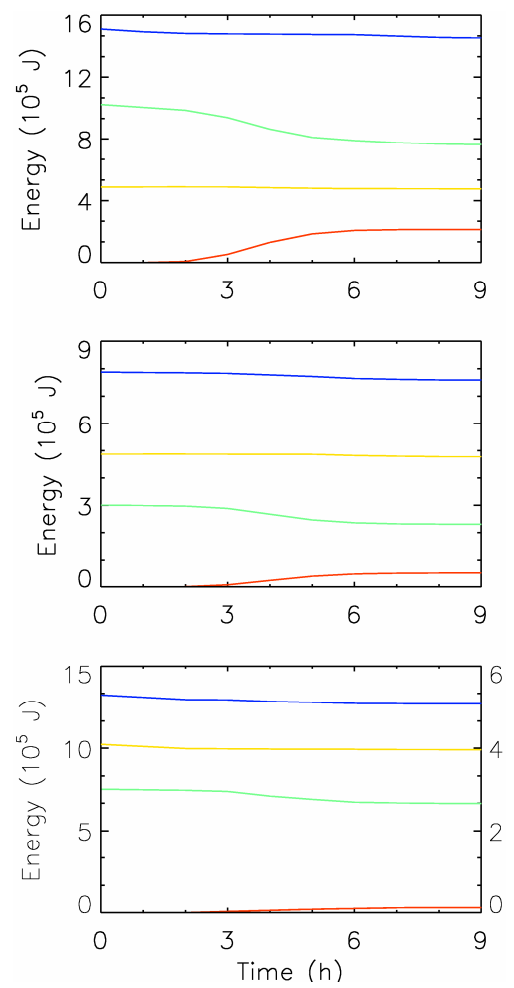

Fig. 3. Evolution of wave energies with time in resonant interactions in cases $1-3$. The green, yellow and red lines denote energies of the primary, secondary and excited waves, respectively; and the blue line denotes their total energy. The top, middle and bottom panels represent the results in cases $1-3$, respectively. Note in bottom panel, the scales for the energies of the primary (green) and excited (red) waves are on the right due to their small values.

and 2. Hence, the sum and difference matching conditions for frequencies cannot be satisfied, which indicates that both the sum and difference resonant interactions do not happen. Since the resonant interaction cannot occur, the third wave would be excited only through the nonresonant interaction if it arose. In the study on the resonant interaction, Huang et al. (2009) introduced a detuning degree of interaction, and explained the new wave excited through the resonant rather than nonresonant interaction. The detuning degree is defined as $\delta=\left|\frac{\Omega\left(\boldsymbol{k}_{1}\right) \pm \Omega\left(\boldsymbol{k}_{2}\right)-\Omega\left(\boldsymbol{k}_{1} \pm \boldsymbol{k}_{2}\right)}{\Omega\left(\boldsymbol{k}_{1} \pm \boldsymbol{k}_{2}\right)}\right|$, which was proposed to measure whether or not there is an effective energy exchange in the nonlinear interactions of gravity waves. If the detuning degree of sum or difference interaction equals to zero $\left(\delta_{\mathrm{s}}=0\right.$ or $\delta_{\mathrm{d}}=0$ ), both the wave vector and frequency matching conditions are satisfied, and the corresponding sum or difference resonant interaction happens, such as $\delta_{\mathrm{s}}=0$ in case 1 and $\delta_{\mathrm{d}}=0$ in cases 2 and 3 . According to the wavenumbers of waves 1 and 2 listed in Table 2, the detuning degrees of sum and difference interactions are calculated to be $\delta_{\mathrm{s}}=0.28$ and $\delta_{\mathrm{d}}=2.17$, respectively. Such a large detuning degree $\left(\delta_{\mathrm{d}}=2.17\right)$ of difference interaction implies that there is no significant energy transfer in the difference nonresonant interaction, however, considering a small value of the detuning degree of sum interaction $\left(\delta_{\mathrm{s}}=0.28\right)$, one can expect that a new wave may be excited through the sum nearresonant interaction. In cases 5 and 6 , the detuning degrees of sum interaction are calculated to be $\delta_{\mathrm{s}}=1.49$ and 0.78 , respectively, while the detuning degrees of difference interaction are $\delta_{\mathrm{d}}=0.24$ and 0.45 , respectively. Therefore, the difference near-resonant interactions are likely to arise in both cases 2 and 3 .

Figure 4 shows the new wave excitations in the second case group (cases 4-6). After 9h, a new wave is generated in each case and the nonlinear interactions are nearly completed. Figure 5 exhibits the normalized wavenumber spectra of these three waves. The dominant wavelengths of the excited waves can be derived from its peak spectrum at $t=9 \mathrm{~h}$. In case 4 , the new wave has a horizontal wavelength of $\lambda_{x}=$ $40 \mathrm{~km}$ just equal to the matching wavelength $\left(L_{s x}=40 \mathrm{~km}\right)$, and a vertical wavelength of $\lambda_{z}=-4.72 \mathrm{~km}$ much shorter than the matching value $\left(L_{s z}=-6.36 \mathrm{~km}\right)$; and its frequency is $21.26 \times 10^{-4} \mathrm{Rad} \mathrm{s}^{-1}$ derived from the dispersion relation, which is slightly larger than the sum $\left(20.59 \times 10^{-4} \mathrm{Rad} \mathrm{s}^{-1}\right)$ of the frequencies of waves 1 and 2 . It can be noted that the wavelengths of wave 3 in Table 2 are intentionally selected to be the same as those of this new wave, thereby, waves 1-3 in Table 2 made up a near-resonant triad. The horizontal and vertical wavelengths of the new waves are 49.66 and $5.35 \mathrm{~km}$ in case 5 and 151.58 and $-2.36 \mathrm{~km}$ in case 6 , respectively. In case 5 , the frequency of the excited wave is $19.42 \times 10^{-4} \mathrm{Rad} \mathrm{s}^{-1}$ quite close to the value $\left(18.72 \times 10^{-4} \operatorname{Rad~s}^{-1}\right)$ of $\omega_{3}-\omega_{2}$, while in case 6 , the excited wave has a frequency of $2.82 \times 10^{-4} \operatorname{Rad~s}^{-1}$, which is smaller than the value $\left(3.21 \times 10^{-4} \mathrm{Rad} \mathrm{s}^{-1}\right)$ of $\omega_{3}-\omega_{1}$. As we expected, the new waves are generated through the sum near-resonant interaction in case 4 and through the difference 

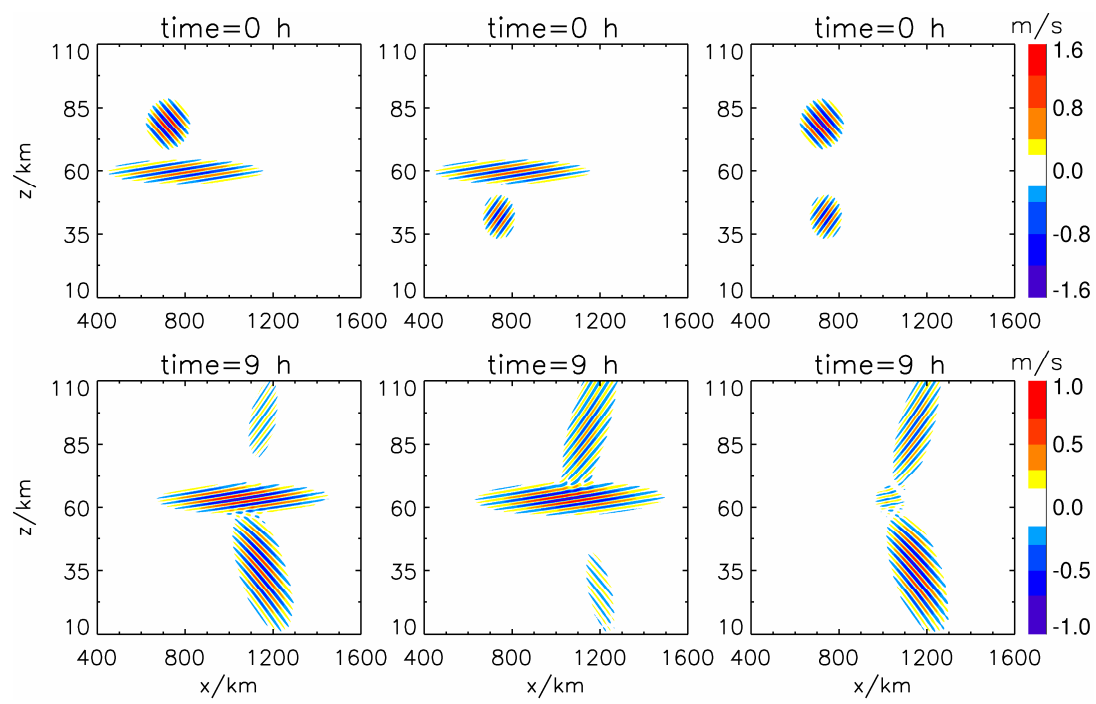

Fig. 4. Sum and difference near-resonant interactions in cases 4-6. The first, secondary and third columns represent results in cases 4-6, respectively.
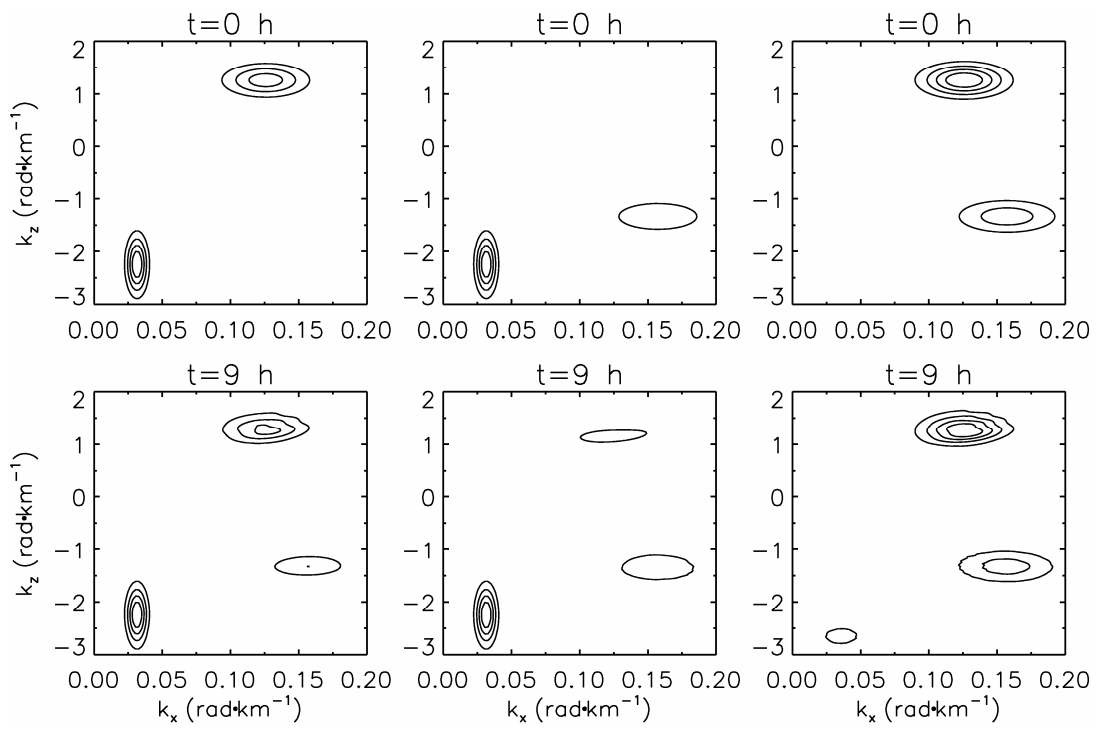

Fig. 5. Similar to Fig. 2, but for cases 4-6.

near-resonant interaction in cases 5 and 6 . An interesting phenomenon is noted that the wavelengths and frequencies of the new waves in cases 5 and 6 are not completely consistent with those of waves 1 and 2 listed in Table 2, respectively. Therefore, for the near-resonant interaction, when the excited wave substitutes for one initial wave, the anew excited wave is not the same as the substituted initial wave, which is different from the reversibility in the resonant interaction. The near-resonant interaction of gravity wave packets shows a characteristic of irreversible excitation.

Figure 6 shows the evolution of wave energies with time in cases 4-6. At the beginning time, the energies of waves $1-3$ are $10.25 \times 10^{5} \mathrm{~J}, 9.24 \times 10^{5} \mathrm{~J}$ and $3.75 \times 10^{5} \mathrm{~J}$, respec- tively. At the time of $9 \mathrm{~h}$, the excited waves obtain the energies of $1.06 \times 10^{5} \mathrm{~J}, 4.65 \times 10^{4} \mathrm{~J}$ and $8.12 \times 10^{3} \mathrm{~J}$ in cases $4-6$ through the near-resonant interactions, respectively. Wave 1 is the primary wave in case 4 and wave 3 plays the primary wave role in cases 5 and 6 , thus, the interaction degrees are calculated to be $10.34 \%, 12.41 \%$ and $2.17 \%$ in cases $4-6$, respectively. In this case group, the total wave energies experience a slight decline with a loss of about $2.1-3.7 \%$ at $t=9 \mathrm{~h}$.

In the third case group (cases 7-9), the initial waves (waves 1 and 2 in case 7, waves 2 and 3 in case 8, and waves 1 and 3 in case 9) are listed in Table 3 . According to the scale of waves 1-3 presented in Table 3, the horizontal and vertical 

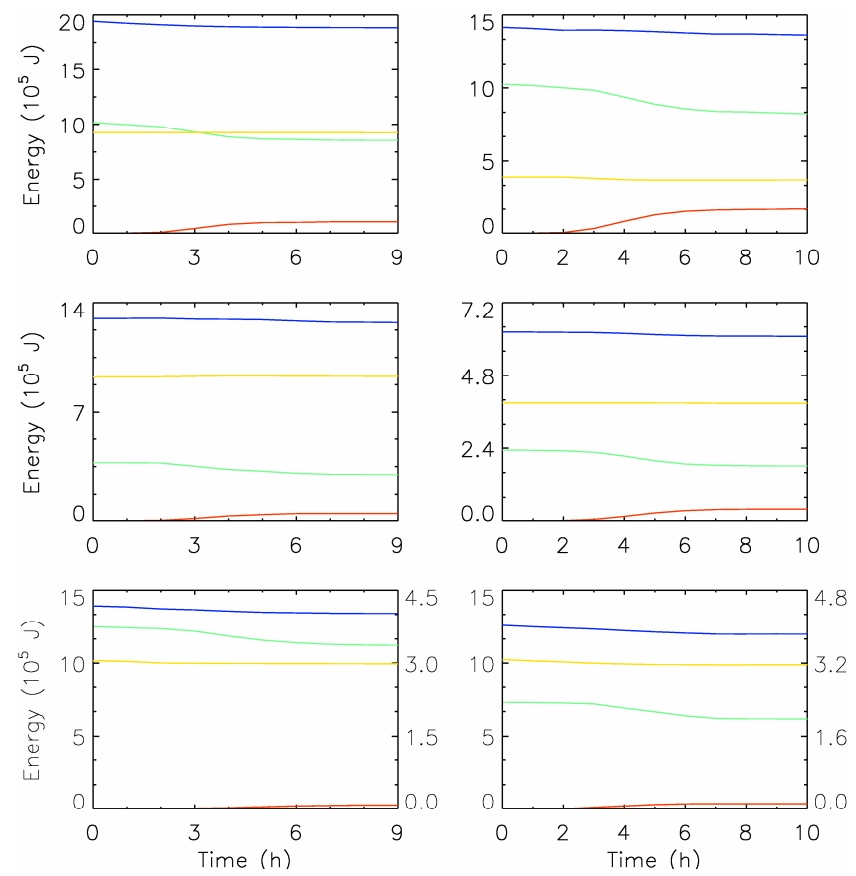

Fig. 6. Similar to Fig. 3, but for cases 4-6 (left columns) and cases 7-9 (right columns). The green, yellow and red lines denote energies of the primary, secondary and excited waves, respectively; and the blue line denotes their total energy.

grid sizes are altered to be $\Delta x=3.5 \mathrm{~km}$ and $\Delta z=0.2 \mathrm{~km}$. In case 7 , the detuning degrees of sum and difference interactions are $\delta_{\mathrm{s}}=0.23$ and $\delta_{\mathrm{d}}=3.97$, respectively, thus, a new wave is excited through the sum near-resonant interaction, which is shown in Fig. 7. Figure 8 shows the normalized wavenumber spectra of the interacting waves. At the time of $10 \mathrm{~h}$, the wavelengths of the excited wave are calculated to be $\lambda_{x}=35.49 \mathrm{~km}$ and $\lambda_{z}=-4.0 \mathrm{~km}$, and its frequency of $\omega=20.32 \times 10^{-4} \mathrm{Rad} \mathrm{s}^{-1}$ is slightly less than the sum $\left(21.08 \times 10^{-4} \mathrm{Rad} \mathrm{s}^{-1}\right)$ of the frequencies of waves 1 and 2. This new wave is chosen to be an initial wave (wave 3) in cases 8 and 9. The detuning degrees of interactions are $\delta_{\mathrm{s}}=1.64$ and $\delta_{\mathrm{d}}=0.20$ in case 8 and $\delta_{\mathrm{s}}=0.70$ and $\delta_{\mathrm{d}}=0.31$ in case 9. Therefore, through the difference near-resonant interaction, a new wave is excited in these two cases, as shown in Fig. 7. According to the normalized wavenumber spectra in Fig. 8, the horizontal and vertical wavelengths of the new wave are 50.40 and $4.41 \mathrm{~km}$ in case 8 and 100.8 and $-2.21 \mathrm{~km}$ in case 9 , respectively. Their frequencies are $15.81 \times 10^{-4} \mathrm{Rad} \mathrm{s}^{-1}$ and $3.98 \times 10^{-4} \mathrm{Rad} \mathrm{s}^{-1}$, which differ from the value $\left(17.29 \times 10^{-4} \operatorname{Rad~s}^{-1}\right)$ of $\omega_{3}-\omega_{2}$, and the value $\left(2.27 \times 10^{-4} \mathrm{Rad} \mathrm{s}^{-1}\right)$ of $\omega_{3}-\omega_{1}$, respectively. Similar to cases 5 and 6 , these two new waves in cases 8 and 9 are not consistent with waves 1 and 2 presented in Table 3 , which shows the irreversible excitation in the near-resonant interaction. This irreversible characteristic can be further clearly confirmed by comparing case 8 with case 5 . We name the new waves in cases 5 and 8 as wave 4 . If the near-resonant excitation also meets the reversibility, the wavelengths and frequencies of waves 4 in cases 5 and 8 should nearly be the same as those of wave 1 (an identical wave), similar to the result in case 2 . However, in fact, because the vertical wavelength $(5.35 \mathrm{~km})$ of wave 4 in case 5 is far larger than that $(4.41 \mathrm{~km})$ in case 8 , the vertical group velocity of wave 4 in case 5 is much faster than that in case 8 , which is evidently seen from their vertical positions in Figs. 4 and 7. The energy centre of the new wave in case 8 is at $z=36.91 \mathrm{~km}$ at $t=10 \mathrm{~h}$, which is higher than that $(z=24.95 \mathrm{~km})$ at $t=9 \mathrm{~h}$ in case 5 and even slightly higher than that $(z=35.85 \mathrm{~km})$ at $t=9 \mathrm{~h}$ in case 2 . The evolution of wave energies of the interacting waves for this case group is also displayed in Fig. 6. At the start time, the energy of the primary wave is $10.25 \times 10^{5} \mathrm{~J}$ in case 7 and $2.34 \times 10^{5} \mathrm{~J}$ in cases 8 and 9 , and at $t=10 \mathrm{~h}$, the exited wave obtains the energy of $1.69 \times 10^{5} \mathrm{~J}, 3.82 \times 10^{4} \mathrm{~J}$ and $1.12 \times 10^{4} \mathrm{~J}$. Hence, the degrees of interaction are calculated to be $16.49 \%, 16.32 \%$ and $4.79 \%$ in cases $7-9$, respectively.

We pay attention to the feature of the spectral energy transfer through the nonlinear interaction. Figures 3 and 6 show that in the three case groups, relative to considerable energy exchange in the first two cases, the energy transfer is quite small in the third case. The interaction of $4.79 \%$ in case 9 $(2.17 \%$ in case 6 and $4.25 \%$ in case 3 ) is obvious less than $16.49 \%$ and $16.32 \%$ in cases 7 and $8(10.34 \%$ and $12.41 \%$ in cases 4 and 5, 20.78\% and $17.04 \%$ in cases 2 and 3). It can also be clearly observed from Figs. 1, 4 and 7 that there are strong excited waves in the first two cases, but a rather weak new wave in the third case. Since this phenomenon occurs in both the resonant and near-resonant interactions, it should be a common characteristic in the nonlinear interaction. By comparing cases 3, 6 and 9 with the other six cases, one can find that the vertical wavelengths and frequencies of the excited waves are much smaller in cases 3, 6 and 9 than in the other cases. For example, in the second case group, cases 4 and 5 have strong excited waves with vertical wavelengths of -4.72 and $5.35 \mathrm{~km}$, however, the vertical wavelength of the weak excited wave in case 6 is the small value of $-2.36 \mathrm{~km}$; correspondingly, the frequencies $\left(21.26 \times 10^{-4} \mathrm{Rad} \mathrm{s}^{-1}\right.$ and $\left.19.42 \times 10^{-4} \mathrm{Rad} \mathrm{s}^{-1}\right)$ of the strong excited waves are almost one-order larger than that $\left(2.82 \times 10^{-4} \mathrm{Rad} \mathrm{s}^{-1}\right)$ of the weak excited wave. Therefore, according to the numerical results, in the nonlinear interactions, the energy transfer to the high frequency excited wave is more efficient than that to the low frequency excited wave, and the nonlinear energy cascade from the large to small vertical scale spectral components is weak through both the resonant and near-resonant interactions.

In the resonant interaction, the interacting three waves obey the matching conditions of wavenumbers and frequencies, as shown in cases 1-3. Here, the matching relation in the near-resonant interaction is examined. Table 4 lists the calculated wavelengths and frequencies of the excited 
Table 3. Initial parameters of waves 1-3 regarded as initial perturbations in cases 7-9.

\begin{tabular}{rrrcrrrrr}
\hline & $\begin{array}{r}\lambda_{x} \\
(\mathrm{~km})\end{array}$ & $\begin{array}{r}\lambda_{z} \\
(\mathrm{~km})\end{array}$ & $\begin{array}{c}k_{x} \\
\left(10^{-4} \mathrm{Rad} \mathrm{m}^{-1}\right)\end{array}$ & $\begin{array}{r}k_{z} \\
\left(10^{-3} \mathrm{Rad} \mathrm{m}^{-1}\right)\end{array}$ & $\begin{array}{r}\omega \\
\left(10^{-4} \mathrm{Rad} \mathrm{s}^{-1}\right)\end{array}$ & $\begin{array}{c}u_{c} \\
\left(\mathrm{~ms}^{-1}\right)\end{array}$ & $\begin{array}{r}x_{c} \\
(\mathrm{~km})\end{array}$ & $\begin{array}{r}z_{c} \\
(\mathrm{~km})\end{array}$ \\
\hline Wave 1 & 50 & 5.0 & 1.26 & 1.26 & 18.05 & 5.0 & 488 & 80 \\
Wave 2 & 120 & -2.0 & 0.52 & -3.14 & 3.03 & 1.0 & 600 & 60 \\
Wave 3 & 35.49 & -4.0 & 1.77 & -1.57 & 20.32 & 0.4 & 526 & 44.4 \\
\hline
\end{tabular}


Fig. 7. Sum and difference near-resonant interactions in cases 7-9. The first, secondary and third columns represent results in cases 7-9, respectively.
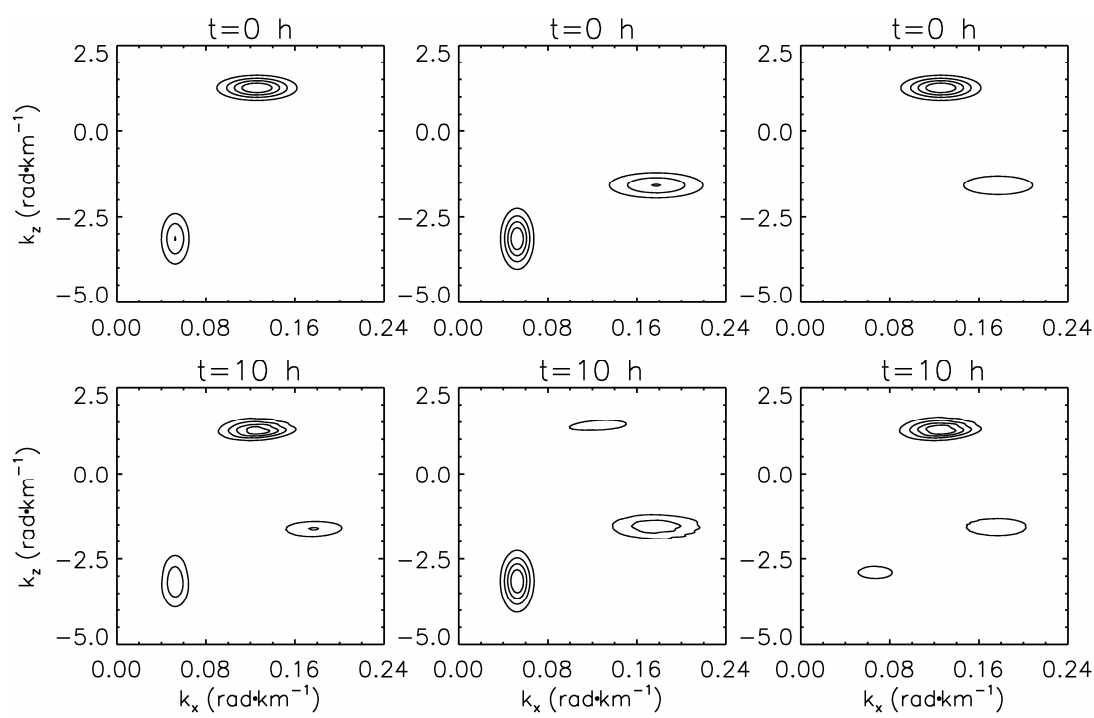

Fig. 8. Similar to Fig. 2, but for cases 7-9. 
Table 4. Comparison between the calculated wavelengths and frequencies of the excited waves and their matching values in cases 4-9.

\begin{tabular}{lrrrrrr}
\hline & $\begin{array}{r}\lambda_{x} \\
(\mathrm{~km})\end{array}$ & $\begin{array}{r}\lambda_{z} \\
(\mathrm{~km})\end{array}$ & $\begin{array}{r}\omega \\
\left(10^{-4} \mathrm{Rad} \mathrm{s}^{-1}\right)\end{array}$ & $\begin{array}{r}L_{x} \\
(\mathrm{~km})\end{array}$ & $\begin{array}{r}L_{z} \\
(\mathrm{~km})\end{array}$ & $\begin{array}{r}\omega_{\mathrm{m}} \\
\left(10^{-4} \mathrm{Rad} \mathrm{s}^{-1}\right)\end{array}$ \\
\hline Case 4 & 40 & -4.72 & 21.26 & 40 & -6.36 & 20.59 \\
Case 5 & 49.66 & 5.35 & 19.42 & 50 & 6.88 & 18.72 \\
Case 6 & 151.58 & -2.36 & 2.82 & 200 & -2.43 & 3.21 \\
Case 7 & 35.49 & -4.0 & 20.32 & 35.29 & -3.33 & 21.08 \\
Case 8 & 50.40 & 4.41 & 15.81 & 50.39 & 4.0 & 17.29 \\
Case 9 & 100.8 & -2.21 & 3.98 & 122.3 & -2.22 & 2.27 \\
\hline
\end{tabular}
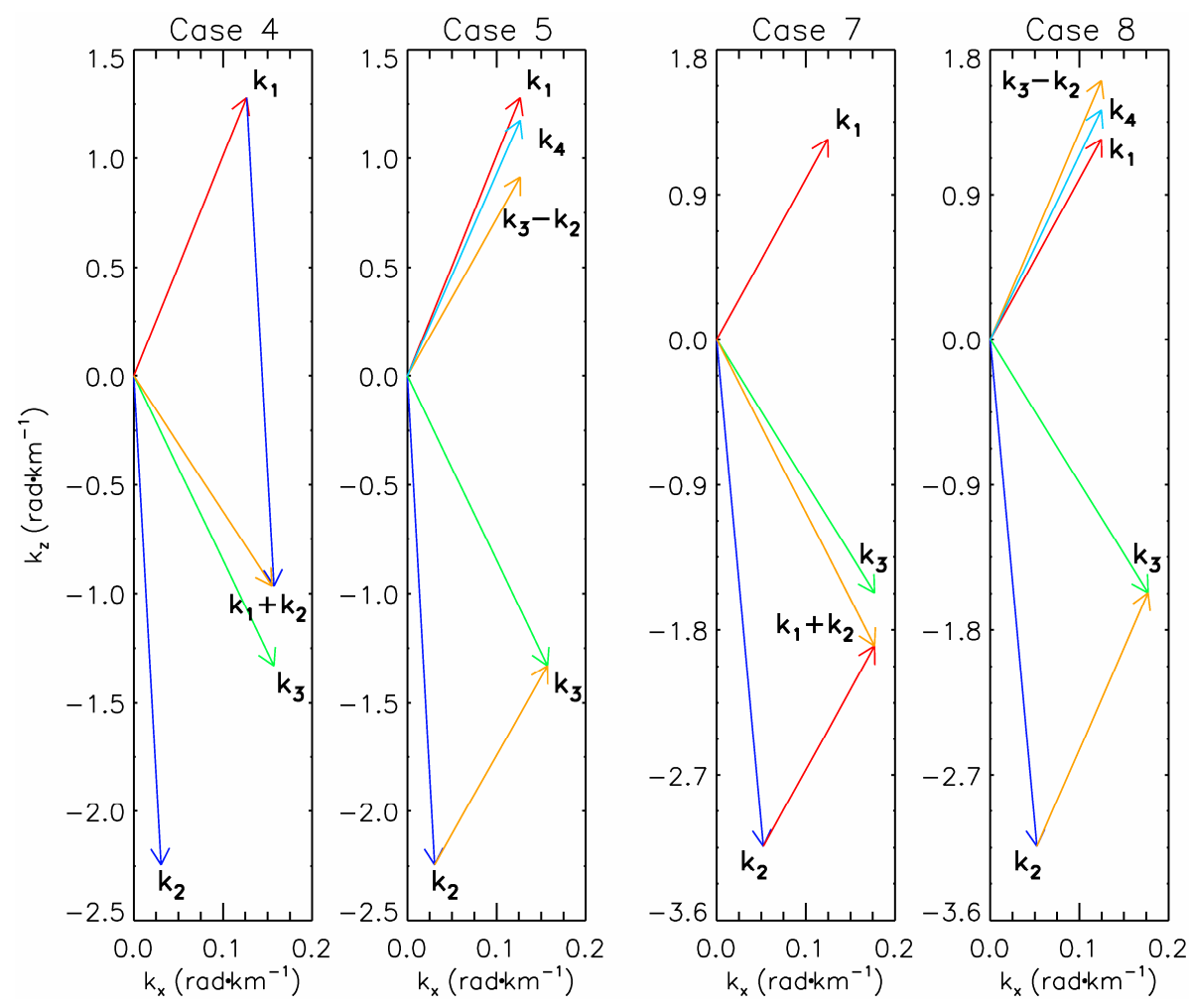

Fig. 9. Wave vectors of interacting waves and matching wave vectors in cases $4,5,7$ and 8 .

waves and the matching values derived from the corresponding matching conditions for cases 4-9. In Table 4, $L_{x}$ and $L_{z}$ denote the matching horizontal and vertical wavelengths, and $\omega_{\mathrm{m}}$ denotes the matching frequency. In case 4 , the excited wave has $\lambda_{z}=-4.72 \mathrm{~km}$ far shorter than $L_{s z}=-6.36 \mathrm{~km}$, but $\lambda_{x}=L_{s x}=40 \mathrm{~km}$. In cases 5,7 and 8 , the horizontal wavelengths of $49.66,35.49$ and $50.40 \mathrm{~km}$ for the excited waves also approximately equal their matching values of 50, 35.29 and $50.39 \mathrm{~km}$, respectively. Figure 9 shows the wave vectors of the interacting waves and the matching wave vectors in the cases 4, 5, 7 and 8. It can be seen from Fig. 9 that in these four cases, the wavenumbers of the interacting waves obvious mismatch in the vertical direction, but approximately match in the horizontal direction. However, for cases 6 and 9 with a weak excited wave, both the hor- izontal and vertical wavenumbers of the interacting waves show moderate mismatch. We can make a conjecture about the approximate match of the horizontal wavenumbers of the interacting waves. If both the horizontal and vertical components of the wave vectors meet the matching conditions in the near-resonant interaction, the frequencies of three waves will mismatch too much, thus, this situation does not benefit the energy exchange to the greatest extent. In order to moderately diminish the mismatching degree of frequencies, the vertical wavenumber of the excited wave may be moderately adjusted because the frequency of gravity wave is more sensitive to the variation of the vertical number than that of the horizontal wavenumber due to the vertical wavenumber generally 1-2 order larger than the horizontal wavenumber, in this case, the horizontal components of wave vectors 
may approximately keep matched. For instance, in case 4, if $\lambda_{x}=L_{s x}=40 \mathrm{~km}$ and $\lambda_{z}=L_{s z}=-6.36 \mathrm{~km}$, the frequency of $28.46 \times 10^{-4} \mathrm{Rad} \mathrm{s}^{-1}$ for the new wave is much larger than the value $\left(20.59 \times 10^{-4} \mathrm{Rad} \mathrm{s}^{-1}\right)$ of $\omega_{1}+\omega_{2}$, thus, the large mismatch in frequency may prevent the wave energy from exchanging as much as possible. The numerical results show the frequency $\left(21.26 \times 10^{-4} \mathrm{Rad} \mathrm{s}^{-1}\right)$ of the excited wave approaching the sum of $\omega_{1}+\omega_{2}$ due to its vertical wavelength smaller than the matching value. In this instance, the considerable energy transfer occurs. However, it is enigmatic that for the nonlinear interaction with a low frequency excited wave with small vertical scale, both the horizontal and vertical wavenumbers of the new wave are moderately adjusted due to the requirement of the energy exchange to the greatest possible degree. With the moderate adjustment of wavenumbers, the frequency mismatch decreases, and it may be possible that the interacting waves show an approximate frequency match. For example, in case 5, the frequency $\left(19.42 \times 10^{-4} \mathrm{Rad} \mathrm{s}^{-1}\right)$ of the excited wave approximately equals the difference $\left(18.72 \times 10^{-4} \mathrm{Rad} \mathrm{s}^{-1}\right)$ of $\omega_{3}-\omega_{2}$.

\section{Summary}

In this paper, the nonlinear interactions of gravity waves are quantitatively studied. By the numerical experiments of resonant and near-resonant triads, we exhibited the excitation of new waves in the sum and difference interactions and discussed the energy exchange among the spectral components. The numerical results show: (1) the energy exchange between two high frequency waves is more efficient than from a high frequency wave to a rather low frequency excited wave. The nonlinear cascade toward increasing vertical wavenumbers is weak through the interaction; (2) the resonant excitation is reversible while the near-resonant excitation is not. Since the interacting waves satisfy the corresponding resonant conditions of wavenumbers and frequencies in the sum and difference resonant interactions, the resonant interactions should exhibit the reversible excitation; and (3) the near-resonant interaction shows a complexity of match relation. There is an interesting result that when the sum and difference near-resonant interactions take place between high and low frequency waves, the wave vectors tend to approximately match in the horizontal direction. However, when a weak wave is excited through the difference interaction between two high frequency waves, their horizontal and vertical wavenumbers show a small mismatch. With the moderate adjustment of the wavenumber of the excited wave, its frequency approaches or even possibly equals its matching value.

Although the wave energy transfer to a high frequency excited wave is intense, this does not mean that a strong new wave with high frequency must be generated through the sum of nonlinear interaction because of the restriction of the detuning degree of interaction. For example, in case 6 , the two high frequency initial waves with $18.05 \times 10^{-4} \mathrm{Rad} \mathrm{s}^{-1}$ and $21.26 \times 10^{-4} \mathrm{Rad} \mathrm{s}^{-1}$ excite only a weak new wave with low frequency of $2.82 \times 10^{-4} \mathrm{Rad} \mathrm{s}^{-1}$ through the difference near-resonant interaction rather than a strong one with frequency approaching their sum $\left(39.31 \times 10^{-4} \operatorname{Rad~s}^{-1}\right)$ through the sum near-resonant interaction due to the large detuning degree of sum interaction. This indicates that for the nonresonant interaction, the energy exchange may be effective in the near-resonant interaction, but insignificant in the far-resonant interaction. If the detuning degrees of both sum and difference interactions are small enough, it might be possible that a strong new wave is generated through the sum interaction and, simultaneously, another weak wave is excited through the difference interaction.

In the nonlinear interactions, the energy exchange between two small vertical wavenumber waves is strong and it is a reversible bidirectional process, as shown in cases 1 and 2, cases 4 and 5, and cases 7 and 8 . However, the energy transfer from small to large vertical wavenumber component is inefficient and it is an irreversible process, such as in cases 3,6 and 9. In this case, the nonlinear spectral energy cascade and dissipation may not be a dominant dissipation mechanism. Because the gravity waves are constrained by the dispersion and polarization relations, their energy cascade is different from the strong and rapid turbulence cascade. If the sum and difference interactions take place among waves with two finite frequency bands around a high and a low frequency, the high frequency band would be spread on both sides by the width of the low frequency band. This effect agrees with the result presented by Broutman et al. (1997), though under a different interaction scenario. Therefore, this nonlinearity may substantially contribute to spectral broadening. In addition, the high frequency waves with large vertical scale can usually permeate the background atmosphere with wind shear and temperature gradient into the middle and upper atmosphere and obtain large amplitudes. Once significant energy exchange arises due to the strong nonlinear interaction, the probability of these waves reaching their instability thresholds decreases, and they may propagate and transport the energy and momentum to much higher altitudes. Hence, the nonlinear energy transfer may play a significant role in limiting wave amplitude growth and energy budget in the low thermosphere. It should be noted that the influence of the earth rotation on the interaction cannot be examined in our two-dimensional model, and the effects of the wind shear and temperature gradient in the realistic background atmosphere still require the great efforts by the theoretical, modelling and experiment studies.

Acknowledgements. The authors would like to thank the editor and anonymous reviewers for their comments on the manuscript. This work was jointly supported by the National Natural Science Foundation of China through grant 41074110, 41174133, 40825013 and 40890160; Ocean Public Welfare Scientific Research Project, State Oceanic Administration People's Republic of China (No. 201005017); the Open Programs of Science and Technology on Electromagnetic Environment Laboratory; the Special Science 
Foundation for New Teacher in University; the Open Programs of Key Laboratory of Geospace Environment; the Fundamental Research Funds for the Central Universities; the China Meteorological Administration grant (No. GYHY201106011). Part of the work was completed during K. M. Huang's visit to Embry-Riddle Aeronautical University, supported by NSF grants ATM-1049451 and ATM-1144343.

Topical Editor C. Jacobi thanks two anonymous referees for their help in evaluating this paper.

\section{References}

Alexander, M. J. and Teitelbaum, H.: Observation and analysis of a large amplitude mountain wave event over the Antarctic peninsula, J. Geophys. Res., 112, D21103, doi:10.1029/2006JD008368, 2007.

Alexander, M. J., Holton, J. R., and Durran, D. R.: The gravity wave response above deep convection in a squall line simulation, J. Atmos. Sci., 52, 22299-22309, 1995.

Axelsson, P., Larsson, J., and Stenflo, L.: Nonlinear interaction between acoustic gravity waves, Ann. Geophys., 14, 304-308, doi:10.1007/s00585-996-0304-3, 1996.

Beard, A. G., Mitchell, N. J., Williams, P. J. S., and Kunitake, M.: Non-linear interactions between tides and planetary waves resulting in periodic tidal variability, J. Atmos. Sol.-Terr. Phy., 61, 363-376, 1999.

Bertherton, F. P.: Resonant interactions between waves: 3. The case of discrete oscillations, J. Fluid Mech., 20, 457-479, 1964.

Broutman, D., Macaskill, C., McIntyre, M. E., and Rottman, J. W.: On Doppler-spreading models of internal waves, Geophys. Res. Lett., 24, 2813-2816, 1997.

Chunchuzov, I. P.: On the nonlinear shaping mechanism for gravity wave spectrum in the atmosphere, Ann. Geophys., 27, 41054124, doi:10.5194/angeo-27-4105-2009, 2009.

Dewan, E. M.: The saturated-cascade model for atmospheric gravity wave spectra, and the wavelength-period (W-P) relations, Geophs. Res. Lett., 21, 817-820, 1994.

Dong, B. and Yeh, K. C.: Resonant and nonresonant wave-wave interactions in an isothermal atmosphere, J. Geophys. Res., 93, 3729-3744, 1988.

Dunkerton, T. J.: Effect of nonlinear instability on gravity-wave momentum transport, J. Atmos. Sci., 44, 3188-3209, 1987.

Dysthe, K. B., Juren, C., and Stenflo, L.: On resonant interactions of atmospheric waves, Phys. Scr., 9, 226-228, 1974.

Fovell, R., Durran, D., Holton, J. R.: Numerical simulations of convectively generated stratospheric gravity waves, J. Atmos. Sci., 49, 1427-1442, 1992.

Fritts, D. C. and Alexander, M. J.: Gravity wave dynamics and effects in the middle atmosphere, Rev. Geophys., 41, 1003, doi:10.1029/2001RG000106, 2003.

Fritts, D. C. and Dunkerton T. J.: Fluxes of heat and constituents due to convectively unstable gravity waves, J. Atmos. Sci., 42, 549-556, 1985.

Fritts, D. C. and Luo, Z.: Gravity wave excitation by geostrophic adjustment of the jet stream, Part I: Two-dimensional forcing, J. Atmos. Sci., 49, 681-697, 1992.

Fritts, D. C., Sun, S. J., and Wang, D. Y.: Wave-wave interactions in compressible atmosphere: 1 . A general formulation including rotation and wind shear, J. Geophys. Res., 97, 9975-9988, 1992.
Hasselmann, K.: Feynman diagrams and interaction rules of wavewave scattering processes, Rev. Geophys., 4, 1-32, 1966.

Hecht, J. H., Walterscheid, R. L., and Vincent, R. A.: Airglow observations of dynamical (wind shear-induced) instabilities over Adelaide, Australia, associated with atmospheric gravity waves, J. Geophys. Res., 106, 28189-28197, 2001.

Hei, H., Tsuda, T., and Hirooka, T.: Characteristics of atmospheric gravity wave activity in the polar regions revealed by GPC radio occultation data with CHAMP, J. Geophys. Res., 113, D04107, doi:10.1029/2007JD008938, 2008.

Hines, C. O.: The saturation of gravity wave in the middle atmosphere. Part II: Development of Doppler-spread theory, J. Atmos. Sci., 48, 1360-1379, 1991.

Hines, C. O.: Theory of the Eulerian tail in the spectra of atmospheric and oceanic internal gravity waves, J. Fluid Mech., 448, 289-313, 2001.

Hirsch, C.: Numerical Computation of Internal and External Flows, John Wiley \& Sons, New York, 1990.

Holton, J. R.: The role of gravity wave induced drag and diffusion in the momentum budget of the mesosphere, J. Atmos. Sci., 39, 791-799, 1982.

$\mathrm{Hu}$, Y. Q. and $\mathrm{Wu}, \mathrm{S}$. T.: A full-implicit-continuous-Eulerian (FICE) scheme for multidimensional transient magnetohydrodynamic (MHD) flows, J. Comput. Phys., 55, 33-64, 1984.

Hu, Y. Q., Habbal, S. R., Chen, Y., and Li, X.: Are coronal holes the only source of fast solar wind at solar minimum?, J. Geophys. Res., 108, A10, doi:10.1029/2002JA009776, 2003.

Huang, K. M., Zhang, S. D., and Yi, F.: A numerical study on nonresonant interactions of gravity waves in a compressible atmosphere, J. Geophys. Res., 112, D11115, doi:10.1029/2006JD007373, 2007.

Huang, K. M., Zhang, S. D., and Yi, F.: Propagation and reflection of gravity waves in a meridionally sheared wind field, J. Geophys. Res., 113, D09106, doi:10.1029/2007JD008877, 2008.

Huang, K. M., Zhang, S. D., and Yi, F.: Gravity wave excitation through resonant interaction in a compressible atmosphere, Geophys. Res. Lett, 36, L01803, doi:10.1029/2008GL035575, 2009.

Inhester, B.: The effect of inhomogeneities on the resonant parameteric interaction of gravity wave in atmosphere, Ann. Geophys., 5, 209-218, 1987.

Klostermeyer, J.: On parametric instabilities of finite amplitude internal gravity waves, J. Fluid Mech., 119, 367-377, 1982.

Klostermeyer, J.: Observations indicating parametric instabilities in internal gravity waves at thermospheric heights, Geophys. Astrophys. Fluid Dyn., 29, 117-138, 1984.

Klostermeyer, J.: On the role of parametric instability of internal gravity waves at atmospheric radar observation, Radio Sci., 25 , 983-995, 1990.

Klostermeyer, J.: Two- and three-dimensional parametric instabilities in finite amplitude internal gravity waves, Geophys. Astrophys. Fluid Dyn., 64, 1-25, 1991.

Kshevetskii, S. P. and Gavrilov, N. M.: Vertical propagation, breaking and effects of nonlinear gravity waves in the atmosphere, J. Atmos. Sol.-Terr. Phy., 67, 1014-1030, 2005.

Lane, T. P., Reeder, M. J., and Clark, T. L.: Numerical modelling of gravity wave generation by deep tropical convection, J. Atmos. Sci., 58, 1249-1274, 2001.

Lindzen, R. S.: Turbulence and stress owing gravity wave and tidal breakdown, J. Geophys. Res., 86, 9707-9714, 1981. 
Liu, A. Z.: Estimate eddy diffusion coefficients from gravity wave vertical momentum and heat fluxes, Geophys. Res. Lett, 36, L08806, doi:10.1029/2009GL037495, 2009.

Liu, A. Z. and Gardner C. S.: Vertical heat and constituent transport in the mesopause region by dissipating gravity waves at Maui, Hawaii $\left(20.7^{\circ} \mathrm{N}\right)$, and Starfire Optical Range, New Mexico ( $35^{\circ}$ N), J. Geophys. Res., 110, D09S13, doi:10.1029/2004JD004965, 2005.

Manson, A. H. and Meek, C. E.: Characteristics of gravity waves (10 min- 6 hour) at Saskatoon $\left(52^{\circ} \mathrm{N}, 107^{\circ} \mathrm{W}\right)$ : Observations by the phase coherent medium frequency radar, J. Geophys. Res., 98, 20357-20367, 1993.

Manson, A. H., Meek, C. E., and Hall, G. E.: Correlations of gravity waves and tides in the mesosphere over Saskatoon, J. Atmos. Sol.-Terr. Phy., 60, 1089-1107, 1998.

McComas, C. H. and Bretherton, F. P.: Resonant interaction of oceanic internal waves, J. Geophys. Res., 83, 1387-1412, 1977.

McComas, C. H. and Müller, P.: Time scales of resonant interactions among oceanic internal waves, J. Phys. Oceanogr., 11, 139147, 1981 .

Müller, P., Holloway, G., Henyey, F., and Pomphrey, N.: Nonlinear interactions among internal gravity waves, Rev. Geophys., 24, 493-536, 1986.

Nekrasov, A. K., Shalimov, S. L., Shukla, P. K., and Stenflo, L.: Nonlinear disturbances in the ionosphere due to acoustic gravity waves, J. Atmos. Terr. Phys., 57, 737-741, 1995.

Olbers, D. J.: Non-linear energy transfer and the energy balance of the internal wave in the deep ocean, J. Fluid Mech., 74, 375-399, 1976.

Pancheva, D.: Non-linear interaction of tides and planetary waves in the mesosphere and lower thermosphere: Observations over Europe, Phys. Chem. Earth, 26, 411-418, 2001.

Phillips, O. M.: On the dynamics of unsteady gravity waves of finite amplitude, J. Fluid Mech., 9, 193-217, 1960.

Rüster, R.: VHF radar observations of nonlinear interactions in the summer polar mesosphere, J. Atmos. Terr. Phys., 56, 1289-1299, 1994.

Rüster, R.: High resolution measurements in the summer polar mesosphere, Geophys. Res. Lett., 24, 1115-1118, 1997.

She, C. Y., Li, T., Collins, R. L., Yuan, T., Williams, B. P., Kawahara, T. D., Vance, L. D., Acott, P., Krueger D. A., Liu, H. L., and Hagan, M. E.: Tidal perturbations and variability in the mesopause region over Fort Collins, CO (41N, 105W): Continuous multi-day temperature and wind lidar observations, Geophys. Res. Lett., 31, L24111, doi:10.1029/2004GL021165, 2004.

Smith, S. A., Fritts, D. C., and VanZandt, T. E.: Evidence for a saturated spectrum of atmospheric gravity waves, J. Atmos. Sci., 44, 1404-1410, 1987.

Stenflo, L.: Resonant three-wave interactions in plasmas, Phys. Scr., T50, 15-19, 1994.

Stenflo, L. and Shukla, P. K.: Nonlinear acoustic-gravity waves, J. Plasma Phys., 75, 841-847, 2009.

Tsuda, T., Kato, S., Yokoi, T., Inoue, T., Yamamoto, M., VanZandt, T. E., Fukao, S., and Sato, T.: Gravity waves in the mesosphere observed with the middle and upper atmosphere radar, Radio Sci., 25, 1005-1018, 1990.

Vadas, S. L. and Liu, H.-L.: Generation of large-scale gravity wave and neutral winds in the thermosphere from the dissipation of convectively generated gravity waves, J. Geophys. Res., 114,
A10310, doi:10.1029/2009JA014108, 2009.

Vanneste, J.: The instability of internal gravity waves to localised disturbances, Ann. Geophys., 13, 196-210, doi:10.1007/s00585995-0196-7, 1995.

Vincent, R. A. and Alexander, M. J.: Gravity waves in the tropical and lower stratosphere: An observational study of seasonal and interannual variability, J. Geophys. Res., 105, 17971-17982, 2000.

Vincent, R. A. and Reid I. M.: HF Doppler measurements of mesospheric momentum fluxes, J. Atmos. Sci., 40, 1321-1333, 1983.

Walterscheid, R. L. and Schubert, G.: Nonlinear evolution of an upward propagation gravity wave: Overturning, convection, transience and turbulence, J. Atmos. Sci., 47, 101-125, 1990.

Weinstock, J.: Theoretical gravity wave spectra in the atmosphere: Strong and weak interactions, Radio Sci., 20, 1295-1300, 1985.

Whitham, G. B.: Linear and Nonlinear Wave, John Wiley, New York, 1974.

Widdel, H. U., Bittner, M., and Hoppe, U. P.: Vertical velocities measured at Biscarrosse $\left(44^{\circ} \mathrm{N}\right)$ and by EISCAT at Tromso $\left(69.6^{\circ} \mathrm{N}\right)$ during the DYANA campaign, J. Atmos. Terr. Phys., 56, 1779-1796, 1994.

Wüst, S. and Bittner, M.: Non-linear resonant wave-wave interaction (triad): Case studies based on rocket data and first application to satellite data, J. Atmos. Sol.-Terr. Phy., 68, 959-976, 2006.

Yeh, K. C. and Dong, B.: The nonlinear interactions of gravity waves with the vortical modes, J. Atmos. Terr. Phys., 51, 45-50, 1989.

Yeh, K. C. and Liu, C. H.: The instability of atmospheric gravity waves through wave-wave interactions, J. Geophys. Res., 86, 9722-9728, 1981.

Yeh, K. C. and Liu, C. H.: Evolution of atmospheric spectrum by processes of wave-wave interactions, Radio. Sci., 20, 1279 1294, 1985.

Yi, F.: Resonant interactions between propagating gravity wave packets, J. Atmos. Sol.-Terr. Phy., 61, 675-691, 1999.

Yi, F. and Xiao, Z.: Evolution of gravity waves through Resonant and onresonant interactions in a dissipative atmosphere, J. Atmos. Sol.-Terr. Phy., 59, 305-317, 1997.

Zhang, S. D. and Yi, F.: A numerical study of nonlinear propagation of a gravity-wave packet in compressible atmosphere, J. Geophys. Res., 104, 14261-14270, 1999.

Zhang, S. D. and Yi, F.: A numerical study on the propagation and evolution of resonant interacting gravity waves, J. Geophys. Res., 109, D24107, doi:10.1029/2004JD004822, 2004.

Zhang, S. D. and Yi, F.: A statistical study of gravity waves from radiosonde observations at Wuhan $\left(30^{\circ} \mathrm{N}, 114^{\circ} \mathrm{E}\right)$ China, Ann. Geophys., 23, 665-673, doi:10.5194/angeo-23-665-2005, 2005.

Zhang, S. D. and Yi, F.: Latitudinal and seasonal variations of inertial gravity wave activity in the lower atmosphere over central China, J. Geophys. Res., 112, D05109, doi:10.1029/2006JD007487, 2007.

Zhang, S. D., Yi, F., Huang, C. M., and Zhou, Q.: Latitudinal and seasonal variations of lower atmospheric inertial gravity wave energy revealed by US radiosonde data, Ann. Geophys., 28, 1065-1074, doi:10.5194/angeo-28-1065-2010, 2010.

Zink, F. and Vincent, R. A.: Wavelet analysis of stratospheric gravity wave packets over Macquarie Island: 1. wave parameters, J. Geophys. Res., 106, 10275-10288, 2001. 